Review article

Central Eur J Paed 2017;13(1):30-41.

DOI $10.5457 / \mathrm{p} 2005-114.167$

\title{
IMMUNOLOGICAL EFFECTS OF ADJUVANTS, THEIR MECHANISMS, AND RELEVANCE TO VACCINE SAFETY
}

\author{
Slavko B. MOJSILOVIĆ \\ Institute for Medical Research \\ University of Belgrade, Belgrade, Serbia
}

Correspondence:
slavko@imi.bg.ac.rs
Tel.: + 381112684484
Fax.: + 381112643691

Received: January 18, 2017

Accepted: January 30, 2017

Key words: Adjuvants • Vaccines • Vaccine safety $\bullet$ Innate immunity.

\begin{abstract}
The purpose of this review is to provide a general overview of adjuvants as immune potentiators, and to offer new insights into the immunological basis and molecular mechanisms of their action. Adjuvants are a key component of many vaccines and their use and development enables many avenues of vaccine design that would otherwise be impossible. Nevertheless, adjuvants are often associated with many safety concerns. Search of available medical literature on adjuvants, vaccines, and their mechanisms of action was performed. Additional articles were identified based on citations in retrieved articles. The main role of adjuvants is to trick the immune system in perceiving vaccine antigen as a serious threat, and thus initiate innate and consecutively adaptive response mechanisms, including long-term immune memory to that antigen. Adjuvants do that by triggering the same evolutionary conserved mechanisms that innate immunity utilizes to detect danger. By inducing innate immune reaction, adjuvants can concurrently provoke some undesirable immune response. However, serious adverse reactions to adjuvanted (as well as nonadjuvanted) vaccines are extremely rare, and there are carefully elaborated regulatory mechanisms to ensure that risks of such adverse reactions are kept at minimum. Conclusion. The use of adjuvants allows a great variety of vaccine designs, enabling the development of safer, more effective, more optimized, and more accessible vaccines than it would be possible without adjuvants. Despite frequent calls to debate, all currently used adjuvanted vaccines have repeatedly demonstrated an excellent safety profile and remain one of the principal tools of science-based medicine in preventing infectious diseases.
\end{abstract}

\section{Introduction}

Since the development of toxoid vaccines in the early $20^{\text {th }}$ century, scientists were searching for a way to enhance immunogenicity of non-living vaccines (1). Some of the first adjuvants discovered back then, on empirical basis of trial and error, are still in widespread use today, but only recently some light on the molecular mechanisms of their action has been shed. Now, with the ever-growing knowledge of vaccine and adjuvant immunology and technology, not only that we have deeper understanding of mechanisms of immune potentiation, but it is possible to rationally design a vaccine/adjuvant to improve the effectiveness, safety and manufacturing process of vaccines, and to tailor a desired response to a vaccine to fit the specific need of a particular population (infants, the elderly, immunocompromised) (2). 
The purpose of this review is to present to the readership with a general overview of adjuvants as immune potentiators, and to offer new insights into the immunological basis and molecular mechanisms of their action. Since there is some controversy about the safety of adjuvanted vaccines and their implication in the development of autoimmune diseases, the last section will address this issue.

\section{Why we need adjuvants}

The essence of active immunization (vaccination) is acquiring long-lasting immunity to a pathogen without getting the disease. The best way to achieve that is to simulate natural infection but with a weakened, attenuated, form of the pathogen (3). However, this is not always possible or practical to do in a convenient and safe way. Cultivation and production of live microorganisms is expensive, requires manipulation with potentially dangerous infectious agents, and there is always a possibility of spontaneous reversal to pathogenic form, resulting in a vaccine-associated disease of the host. In such cases, the dead pathogen is used, as a whole organism, or some of its key antigenic subunits. However, since dead organisms or their parts can obviously cause no infection, they may not induce a strong immune response. Hence such preparations are often not sufficiently immunogenic, i.e., they do not confer adequate and long-lasting immunity to the pathogen. Adjuvants (from Latin adjuvareto help) are therefore coadministered to enhance the immunogenicity of the vaccine antigen. To achieve that, a good adjuvant needs to trick the immune system to perceive the vaccine antigen as a serious threat, and thus initiate innate and consecutively adaptive response mechanisms to eliminate the threat and prepare itself (through long-term immune memory) for the next encounter with the same threat (4).
Some inactivated whole-pathogen vaccines do not need an adjuvant since they contain a heterogeneous mixture of diverse antigens and other components of the pathogen that act as intrinsic adjuvants. Also, carbohydrate antigens do not need an adjuvant, as they directly bind to and activate B cells (T-cell-independent activation) (5). However, this type of humoral response is inherently weaker, and, more importantly, of short duration, with no affinity maturation and no immunological memory. For that reason, conjugate vaccines were developed, consisting of carbohydrates conjugated to a protein that provides $\mathrm{T}$ cell epitopes for a T-cell-dependent response needed for antibody class switching, affinity maturation, and longterm immunological memory. Some of the protein components of conjugate vaccines can also have adjuvant capacity (6).

Practical goals of using adjuvants include: increasing the functional titer of antibodies raised against the target antigen; decreasing the dose of antigen needed to elicit immune reaction (antigen sparing); decreasing the number of doses needed to achieve complete immunization (dose reduction); enhancing immune responses in the young, elderly and immunocompromised populations (poor responders); increasing the duration of the vaccine-specific protective response; decreasing the time needed to achieve protective immunity; induction of potent cell-mediated immunity; balancing or redirecting the immune response toward the Th1, Th2, or Th17 arm of the $\mathrm{T}$ cell response, as required; inducing mucosal immunity; and inducing a broader immune response (cross-protection against related strains of pathogen not included in the vaccine) $(7,8)$. In addition to achieving quantitative and qualitative alterations of the immune response, some of these goals, like antigen sparing and dose reduction, have important implications for improving global vaccine supply. Furthermore, by ensuring 
enough immunogenicity to limited number of purified antigens in subunit vaccines, adjuvants contribute to general safety profile of the vaccines (9). In view of the above, it is easy to see why adjuvants are a key component of many vaccines, as well as how their continuing use and development enables many avenues of vaccine design that would otherwise be completely impossible.

\section{How adjuvants work}

In 1926, French veterinarian Gaston Ramon reported that by injecting starch, breadcrumbs, or tapioca, he managed to induce sterile abscesses at the site of injection with an inactivated toxin, which led to increased anti-sera production, confirming the hypothesis that substances able to induce local inflammation at the injection site were also able to enhance anti-sera yield (1). A few years later, British scientist Alexander Glenny discovered the immune-enhancing effects of aluminium salts (8). Aluminium was first used in human vaccines in 1932 and remained the only adjuvant in use in licensed human vaccines for approximately 70 years. Those and other early adjuvants have all been empirically identified for their ability to enhance the adaptive immune responses to a coadministered antigen in experimental models, and their mechanisms of action have long been "the immunologist's dirty little secret" (10).

With the growing understanding of innate immune system and its role in activation and modulation of adaptive immune response, the mechanisms of action of adjuvants are also being unveiled.

\section{The signals of danger}

The key event in the immune response is detecting an antigen in the context of danger. The concept of danger, i.e., specific compo- nents of damaged host tissues (damaged self) as primary signals for activation of protective immunity, as opposed to foreign or non-self structures, was proposed by Polly Matzinger in 1994 (11). Now it is recognized that these are not conflicting, but rather complementary hypotheses. Innate immune system, through a variety of conserved germlineencoded innate receptors, recognizes specific molecular patterns of both exogenous and endogenous origin (12). Components of bacterial or fungal wall, or bacterial and viral nucleic acids, represent examples of pathogenassociated molecular patterns (PAMP). On the other hand, cellular components exposed during cell damage, like adenosine triphosphate (ATP), uric acid, and high mobility group box 1 (HMGB1), represent endogenous damage-associated molecular patterns (DAMP). Sensing danger is the initial step in the activation of innate immunity, and innate immune reaction is the prerequisite for the activation of adaptive immunity and generation of an immunological memory.

\section{Innate receptors}

The most abundant and diverse innate receptors are pattern-recognition receptors (PRR), which comprise Toll-like receptors (TLR), nucleotide oligomerization domain (NOD)like receptors (NLR), and the retinoic acid-inducible gene-I (RIG-I)-like receptors (RLR) $(12,13)$. Each type of PRR has specific ligand(s) and specific signaling pathway. They are strategically located inside the cell or on its surface, depending on the accessibility of their ligands. Ligation of the PRR initiates a signaling network in host cells that regulates transcription of pro-inflammatory genes and coordinates an appropriate immune response to respond to the detected threat.

The best studied of the PRR are TLR. Originally discovered for its contribution to dorso-ventral patterning in Drosophila (fruit 
fly) embryos, Toll molecule is later recognized for its role in the fly's innate immune response to microbial infection (14). There are 10 types of its analogues in humans (TLR1 to TLR10), and much more in other vertebrates (23 different TLR in total), and each of them has specificity for different PAMP. Ligands for TLR include many microbial products, such as lipopolysaccharide (LPS) of Gram-negative bacteria, double-stranded (ds) RNA present in certain viruses, virusspecific single-stranded (ss)RNA sequences, unmethylated $\mathrm{CpG}$ oligonucleotides derived from frequently found motifs in bacterial DNA, flagellin (a bacterial protein), mannan and related compounds of fungal cell walls, and glycophosphoinositol (GPI) anchors of protozoal cell membranes, to name but a few.

NOD-like receptors, such as NLRP3 and NLRP4, upon ligation, form proteolytic complexes called inflammasomes, which cleave the inactive form of pro-inflammatory cytokines IL-1, IL-18 and IL-33 and thus activate them (15). Evidence is steadily accumulating in support of the concept that these and other inflammasomes are key initiators of immune responses, and most likely to be involved in processes governing the selection between activation (immunogenicity, inflammatory response) and inhibition (tolerogenicity), the latter being crucial to immune (and overall) homeostasis. RLR are somewhat less studied than TLR and NLR. It is known that these PRR detect viral replication by binding dsRNA in the cytoplasm of infected cells (16).

\section{Cells expressing PRR}

The central elements in the conveyance of information from innate to adaptive immune systems are antigen-presenting cells (APC) (17). They have the potential to uptake antigens, process them into small peptides and load them onto major histocompatibility complex (MHC) class II molecules for presentation to $\mathrm{T}$ cells. Upon activation, APC upregulate MHC class II, as well as costimulatory molecules (e.g. CD80 and CD86) that provide "signal 2", which is necessary for full activation of $\mathrm{T}$ cells. This second signal assures that only APC that are activated by danger signals can trigger $\mathrm{T}$ cell response (18). The principal APC are dendritic cells (DC). DC are most potent in activating naïve $T$ cells and cross-presenting exogenous antigens through MHC class I pathway to cytotoxic $T$ lymphocytes (CTL). In addition to DC, APC include macrophages, B lymphocytes, activated endothelial and some epithelial cells, as well as some other cell types under certain conditions (19). However, most of these cells lack the full set of costimulatory molecules required to induce the full spectrum of T cell responses. Apart from APC, there are also several cell types not usually involved in antigen-presentation that nevertheless express PRR and thus may contribute to immune responses elicited by adjuvants (20). The exact roles of these cell types in immune responses are still a subject of research.

\section{Mechanisms of action of adjuvants}

Among the first acknowledged mechanisms of adjuvant action are depot effect and antigen delivery. Aluminium salts were considered to function by formation of a depot from which the antigen is slowly released. This was disputed in several studies showing that adsorption to aluminium salts does not increase significantly the half-life of antigen in vivo (21). However, by converting soluble antigen into a particulate form, aluminium adsorption increases antigen uptake by APC, and thus acts as an atigen delivery system. Other adjuvants, classified as delivery systems (liposomes, immune-stimulating complexes, nanoparticles) also function primarily 
as vehicles to deliver antigens to particular cells or cellular compartments (22).

The most general mechanism of adjuvanticity is recruitment of inflammatory cells and establishment of a local immunocompetent environment (20). Virtually all adjuvants induce some level of inflammatory reaction with secretion of proinflammatory cytokines and chemokines, thus recruiting and activating inflammatory cells. This is the central mechanisms of action of emulsions and mineral salts (23). Cells that are damaged in this inflammatory process release DAMP that activate APC and other inflammatory cells.

It is now well established that aluminium salts directly activate NLRP3, member of NLR family, which leads to the formation of inflammasome, and subsequent processing and release of proinflammatory cytokines IL$1 \beta$, IL-18 and IL-33 (24). The same mechanism is demonstrated for some other particulate adjuvants (25). This process is also activated by some DAMP molecules, like ATP, uric acid and monosodium urate crystals. It is postulated that aluminium salts, in addition to direct activation of NLRP3, indirectly activate this pathway by releasing uric acid from necrotic cells (26).

Some adjuvants consist of microbial components or their analogues, and target APC and other inflammatory cells by directly activating TLR and other PRR (7). Activation of APC, more specifically DC, either directly or indirectly, is the central event in the mechanism of action of most adjuvants $(7,20,27)$. Activation of DC leads to the upregulation of cytokines, chemokine receptors, MHC class II, and costimulatory molecules, and to their migration to the $\mathrm{T}$ cell zone of regional lymph nodes, where they present antigens they have sampled to naïve $T$ cells, thus activating them and initiating the adaptive immune response.

\section{The main classes of adjuvants in use}

\section{Mineral salts}

By far the most extensively used vaccine adjuvants today, as has been the case throughout the history of adjuvant use, are aluminium salts. Modern human vaccines use pre-formed aluminium gels containing sulfate, phosphate or hydroxide salts of aluminum, but composition and higher order structure of different formulations varies considerably $(8,22)$. For some proprietary aluminum salt adjuvants, the full chemical formula and process of formulation are not publicly available. Frequently, term "alum" is wrongly used as a catch-all term for aluminum salt-containing adjuvants. Alum is chemically defined as hydrated potassium aluminum sulfate $\left(\mathrm{KAl}_{2}\left(\mathrm{SO}_{4}\right)_{2} \cdot 12 \mathrm{H}_{2} \mathrm{O}\right)$, and it has been used as a reagent in the process of preparing aluminium-based adjuvants, not as an adjuvant per se (28). It is important to acknowledge that surface chemistry, particle size, and structure are important attributes of aluminium gels that influence their properties as adjuvants (29). Aluminium hydroxide and aluminium phosphate have opposite electrical charges at neutral $\mathrm{pH}$. Hence, it is important to carefully select the type of aluminium adjuvant and optimize the conditions of adsorption for each antigen. By simply changing the surface chemistry of aluminium gels by the addition of a defined amount of phosphate or hydroxyl ions, it is possible to alter antigenbinding affinity, stability and potency. The size of particles also influences how innate immunity will react, and the effect can vary from no proinflammatory response, to functional adjuvanticity, to frustrated phagocytosis (8).

With almost a century of experience, aluminum salt adjuvants have demonstrated effective induction of relatively long-lasting protective immunity to extracellular pathogens, ease of formulation, and a long record of general safety (30). They elicit strong Th2 polarization and induce high IgG titers. On 
the other hand, aluminium salts do not stimulate cell-mediated cytotoxic $\mathrm{CD}^{+} \mathrm{T}$ cell response, which is necessary for protection against viruses and other intracellular pathogens $(27,30)$.

\section{Emulsions}

Another classical adjuvant, complete Freund's adjuvant (CFA), discovered by Jules Freund in 1951, is an emulsion of soluble antigen in paraffin oil (water-in-oil emulsion), with a surfactant and inactivated dry Mycobacteria. Because of its high potency, it was long used for induction of immune response in experimental settings, but it was too toxic for use in humans. A less potent variant, and easier for preparation and application, is oil-in-water emulsion called incomplete Freund's adjuvant (IFA), for it does not contain Mycobacteria. However, it is still too reactogenic to be used in human vaccines (7).

There are now two licensed oil-in-water emulsion adjuvants for use in human vaccines - MF59 (Novartis) and AS03 (GlaxoSmithKline). They are both based on squalene as the oil component. Squalene is a biodegradable oil extracted from shark liver, which is also naturally found in human tissues. In addition, MF59 contains surfactants, polyoxyethylene sorbitan mono-oleate (Tween 80) and sorbitan trioleate, and AS03 contains Tween 80 and $\alpha$-tocopherol (vitamin E). They are used in seasonal and pandemic flu vaccines, since they elicit a high titer of neutralizing antibodies, as well as a strong $\mathrm{CD}^{+} \mathrm{T}$ cell response. Both emulsions have good safety records (see below), permit fewer doses and antigen dose sparing, and generate marked memory responses, with a mixed Th1 and Th2 cell phenotypes (31).

\section{TLR-agonists}

Since the discovery of TLR and their fundamental role in innate immunity, much of the effort is directed towards development of adjuvants that would directly target these PRR. Up to now, only one TLR agonist adjuvant has been licensed, but many are in some phase of preclinical research or clinical trials. Monophosphoryl lipid A (MPL) is a derivative of LPS, a TLR 4 agonist, but it is 10,000 times better tolerated than the original lipid A $(2,32)$. Adhered to aluminum salt in AS04 combined adjuvant, it is currently approved for use in one of the vaccines against human papilomavirus (Cervarix ${ }^{\circledR}$ ). It has been shown to be able to increase antibody titers and to induce a strong cellular immune response.

Synthetic analogues of bacterial and viral nucleic acids are also promising candidates for adjuvants. Synthetic 5'-cytosinephosphate-guanine-3' oligodeoxynucelotides (CpG-ODN) that contain unmethylated $\mathrm{CpG}$, characteristic for bacterial DNA, are ligands for TLR9. CpG-ODN enhance antibody responses and strongly polarize Th cell responses towards Th1 and away from Th2 (33). Another synthetic analogue of nucleic acid, polyinosinic:policytydilic acid (poly I:C), has a structure similar to dsRNA, which is unique to some viruses. This analogue binds two types of PRR - TLR3 and MDA5 (a RLR). Activation of both receptors optimizes the magnitude and durability of Th1 cell immunity and $\mathrm{CD}^{+} \mathrm{T}$ cell immunity compared to either pathway alone (34).

Usual ligands for TLR7 and TLR8 are guanosine- and uridine-rich ssRNA. However, ssRNA is very unstable, so other synthetic agonists are used to stimulate these receptors, such as imiquimod and resiquimod (35). Adjuvanticity of these small molecules is substantially improved when they are coupled to an antigen, compared to when they are simply admixed. Bacterial flagellin, a TLR5 agonist, can also be used as an adjuvant, either in a mixture with an antigen or when fused with it as a single recombinant protein (36). Unlike much of the other TLR agonists 
that strongly polarize Th1 cell response, flagellin tends to produce mixed Th1 and Th2 cell responses.

\section{Delivery systems}

Special form of vaccine/adjuvant formulations are delivery systems. Their function is to deliver the antigen into an APC for enhanced processing and presentation. Delivery systems can incorporate in their structure some of the previously mentioned adjuvants. They include liposomes, immune-stimulating complexes and nanoparticles (22).

Liposomes are bilayered or multilayered biodegradable vesicles, made of phospholipids that mimic the structure of natural lipid bilayer membranes, enabling them to enter APC by endocytosis. They are able to carry insoluble antigens and considerably enhance the immunogenicity of weakly immunogenic protein antigens (37). Immune-stimulating complexes (ISCOM) are proprietary forms of liposomes made of phospholipids, cholesterol, and saponins from the bark of Quillaja saponaria, that form cage-like structures (ISCOMATRIX) into which antigens can be entrapped or intercalated (38). They can provide a depot function, as well as facilitated delivery, uptake, and processing of vaccine antigens by APC.

Application of nanotechnology for designing carrier systems for antigen delivery is a new and perspective area of vaccine development (2). These nanovaccines can vary in size, shape, composition, and surface properties, and can be customized to modulate antigen processing and presentation pathways according to specific needs. Rationally developed nanovaccines are one of the most promising candidates for the future of vaccine development.

\section{Safety of adjuvants}

Since adjuvants, by definition, are always administered as a part of a vaccine, and are eval- uated as such, the first challenge in assessing adverse reaction to adjuvants is to separate them from reactions to other vaccine components (39). Bearing in mind that vaccines are generally administered to healthy people, including vulnerable populations such as children and pregnant women, vaccines must meet much higher safety standards than other medicines. As with other medicines, vaccines also have to pass three phases of clinical studies, where safety and efficacy of the vaccine is evaluated on progressively larger and more diverse populations. In order to obtain licensure, a new vaccine has to prove to have a good benefit-to-risk ratio, that is, to demonstrate that its expected benefits in preventing disease clearly outweighs any potential risk to the targeted population. After the licensure, the safety and benefit-to-risk assessments continue indefinitely. Whenever the evidence of causal association between the vaccine and adverse event arise, or the vaccine eventually shows poor efficacy, the benefit-to-risk ratio decreases and the vaccine may be withdrawn.

In view of the discussed roles of adjuvants as immune potentiators, it is not without grounds to presume that adjuvants in vaccines can also provoke an undesirable immune response. Indeed, many adverse events of vaccines can be attributed to adjuvants and their mechanisms of action (40). Adjuvants are generally considered principally responsible for the usual mild to moderate vaccine reactions (redness and swelling at the injection site, low-grade fever, fatigue, myalgia, headache), since these reactions are mostly caused by the activation of innate immunity, with brief release of inflammatory mediators. More serious adverse reactions to adjuvants have been repeatedly found to be rare (30, $31,41,42)$. However, some adjuvants have tentatively been linked to pathological conditions. Some well known examples will be reviewed here. 


\section{Narcolepsy}

During the 2009 H1N1 influenza pandemic in some European countries a number of cases of narcolepsy were reported following vaccination with Pandemrix, a GlaxoSmithKline's pandemic influenza vaccine $(\mathrm{H} 1 \mathrm{~N} 1$ 2009), containing AS03 as adjuvant (43). Narcolepsy is a chronic neurological disorder presenting with excessive daytime sleepiness and often cataplexy - a transient loss of muscle tone triggered by strong emotional stimuli. It is characterized by loss of hypothalamic hypocretin-producing neurons, and is strongly associated with the HLADQB $1{ }^{*} 0602$ genotype. At the same time, no association with narcolepsy has been reported for another similar pandemic flu vaccine, Novartis’ MF59-adjuvanted Focetria ${ }^{\circledR}$. This fact has brought into focus the role of $\mathrm{H} 1 \mathrm{~N} 1$ viral proteins or the AS03 adjuvant as environmental triggers in genetically predisposed persons. It was proposed that $\alpha$-tocopherol, the specific component of AS03, via transcription factor $\mathrm{Nrf} 2$, affects the expression and turnover of hypocretin, leading to an increased formation of longer hypocretinderived fragments that can be presented by HLA-subtype $\mathrm{DQB}^{*} 0602$ (44). Another study, however, suggested a different mechanism (45). It demonstrated increased frequency of antibodies to hypocretin receptor 2 in the sera of narcoleptic patients with a history of immunization by Pandemrix ${ }^{\circledR}$, which cross-reacted with a fragment of influenza nucleoprotein A that was found in Pandemrix vaccine in much greater amount than in other related pandemic vaccines. This antigen-mimicry may also explain the association of cases of narcolepsy with seasonal influenza infections (46). Considering all the data collected from epidemiological studies, as well as experimental research of immunological basis of pandemic influenza vaccineinduced narcolepsy, European Medicines Agency has concluded that the benefit-risk profile of H1N1 2009/AS03 remains favorable (although "the marketing authorization for Pandemrix ${ }^{\circledR}$ has expired following the marketing-authorization holder's decision not to apply for a renewal" in 2016) (47).

\section{Macrophagic myofasciitis}

Macrophagic myofasciitis (MMF) represents a histological lesion containing aluminium crystal-laden macrophages gathered around the muscular fibers, found at the injection site of aluminium-adjuvanted vaccines for a very long time after vaccination (48). A causal association of these lesions with a group of systemic manifestations that includes myalgias, arthralgias, asthenia, muscle weakness, chronic fatigue, and fever, was proposed to represent a distinct immune-mediated condition triggered by aluminum-containing vaccines in genetically susceptible people (49). Some studies suggested that increased level of CCL2 chemokine present in patients with this condition is associated with an impaired capacity to excrete aluminium, which could provide a biological explanation for susceptibility of some individuals to aluminiumbased adjuvants (50). Although this finding certainly merits further investigation, conclusive evidence of a link between aluminium adjuvant-containing vaccines or MMF lesion and systemic muscular disease is still missing, and WHO Global Advisory Committee on Vaccine Safety states that there is no reason to conclude that a health risk exists as a result of administration of aluminium containing vaccines (51).

\section{Squalene and the Gulf War Syndrome}

One of more controversial associations of vaccine adjuvants with an illness is the allegation that squalene is a causative agent of a group of nonspecific systemic symptoms that occurred in veterans of the 1991 Persian Gulf 
War, known as Gulf War syndrome (GWS). The syndrome encompasses various physical, psychical, and cognitive symptoms, and clinically overlaps with post-traumatic stress disorder, chronic fatigue syndrome, fibromyalgia, and other functional disorders. Since the soldiers had been exposed to unique hazardous environmental conditions, many causal factors have been proposed, including vaccinations (52). One study showed a connection between the anti-squalene antibodies and GWS (53). Squalene-based adjuvant was allegedly used in antrax vaccine that was administered to the soldiers, and antibodies to squalene were detected in the sera of most patients affected. However, the initial conclusion was refuted by later studies that found no association between squalene antibody status and chronic multisymptomatic illness, and showed that squalene was not even present in vaccines administered to these soldiers (54). Additionally, squalene per se is poorly immunogenic, it is widespread in nature, even produced in human liver and skin, and low titers of anti-squalene antibodies are routinely found in healthy individuals, and their titer is not increased after administration of squalene-containing vaccines (55).

\section{Autoimmune/Inflammatory Syndrome Induced by Adjuvants}

Many more immune-mediated diseases and conditions are occasionally reported to be associated with adjuvants and vaccines as a whole (56). As a way of grouping together a range of emerging autoimmune diseases with possible adjuvant-associated causes, Yehuda Shoenfeld and Nancy Agmon-Levin proposed a new syndrome - Autoimmune/ Inflammatory Syndrome Induced by Adjuvants (ASIA), also known as Shoenfeld's syndrome (57). In a systematic review published in 2015, Hawkes et al. questioned the clinical value of ASIA, as its diagnostic criteria are very broad and lack a precise definition of temporal association and dosage limits, but recognized that it provides a framework facilitating the investigation of rare and unusual cases of potential vaccine/adjuvant-induced autoimmunity (58).

The occurrence of these rare adverse reactions should by no means hamper further development of new adjuvants or imply that it may be safer not to vaccinate, foregoing protection offered by vaccines. The positive effect on reduction of infectious disease morbidity and mortality, by far exceeds the negative side effects of vaccination. Moreover, one must not overlook the fact that vaccines only mimic natural infections, and infectious agents themselves can elicit the same immune phenomena. Indeed, the risk of developing immune-mediated diseases by acquiring natural infection is even greater than the risk of the same diseases to develop by vaccineassociated reactions. By carefully monitoring the rare adverse events and scrupulously studying their mechanism of development, regulatory agencies, vaccine manufacturers, and researchers are participating in a joint endeavor to identify the specific factors that contribute to these events and to develop even safer vaccines.

\section{Conclusion}

Adjuvants are an extremely important class of vaccine components. Their use allows a great variety of vaccine designs, enabling the development of safe and effective vaccines to protect from many conditions that would otherwise be impossible to create. Although, as vaccines themselves, adjuvants are not riskfree in an absolute sense, they have actually made vaccination safer as well as more effective, since adjuvanted vaccines can often be more finely tuned to specific needs of eliciting an immune response against a particular pathogen, as well as optimized beyond the 
level possible without adjuvants, including the possibility to restrict the number of antigens present in a vaccine, and thus further reduce any risk of undesired (cross-reactive) immune responses to self tissues. Adjuvants can also increase the cost-effectiveness and simplify the logistics of vaccine use. Despite frequent calls to debate, all currently used adjuvants have repeatedly demonstrated an excellent safety profile, and remain, together with the vaccines they are part of, one of the principal tools of science-based medicine in preventing infectious diseases that have cost mankind many lives in the past, and are still threatening to do so wherever and whenever vaccination efforts fall below adequacy.

Acknowledgement: The author wishes to thank Dr. Srđa Janković for his valuable comments and helpful discussion.

Conflict of interest: The author declares that he has no conflict of interest.

\section{References}

1. Di Pasquale A, Preiss S, Tavares Da Silva F, Garçon N. Vaccine Adjuvants: from 1920 to 2015 and Beyond. Vaccines (Basel). 2015;3(2):320-43.

2. Karch CP, Burkhard P. Vaccine technologies: From whole organisms to rationally designed protein assemblies. Biochem Pharmacol. 2016;120:1-14.

3. Hajj Hussein I, Chams N, Chams S, El Sayegh S, Badran R, Raad M, et al. Vaccines Through Centuries: Major Cornerstones of Global Health. Front Public Health. 2015;3:269.

4. Bendelac A, Medzhitov R. Adjuvants of immunity: harnessing innate immunity to promote adaptive immunity. J Exp Med. 2002;195(5):F19-23.

5. Vos Q, Lees A, Wu ZQ, Snapper CM, Mond JJ. B-cell activation by T-cell-independent type 2 antigens as an integral part of the humoral immune response to pathogenic microorganisms. Immunol Rev. 2000;176:154-70.

6. Latz E, Franko J, Golenbock DT, Schreiber JR. Haemophilus influenzae type b-outer membrane protein complex glycoconjugate vaccine induces cytokine production by engaging human Toll-like receptor 2 (TLR2) and requires the presence of
TLR2 for optimal immunogenicity. J Immunol. 2004;172(4):2431-8.

7. Coffman RL, Sher A, Seder RA. Vaccine adjuvants: putting innate immunity to work. Immunity. 2010;33(4):492-503.

8. Powell BS, Andrianov AK, Fusco PC. Polyionic vaccine adjuvants: another look at aluminum salts and polyelectrolytes. Clin Exp Vaccine Res. 2015;4:23-45

9. Pichichero ME, Deloria MA, Rennels MB, Anderson EL, Edwards KM, Decker MD, et al. A safety and immunogenicity comparison of 12 acellular pertussis vaccines and one whole-cell pertussis vaccine given as a fourth dose in 15- to 20-month-old children. Pediatrics. 1997;100(5):772-88.

10. Gayed PM. Toward a modern synthesis of immunity: Charles A. Janeway Jr. and the immunologist's dirty little secret. Yale J Biol Med. 2011;84(2):1318 .

11. Matzinger P. Tolerance, danger, and the extended family. Annu Rev Immunol. 1994;12:991-1045.

12. Takeuchi O, Akira S. Pattern recognition receptors and inflammation. Cell. 2010;140(6):805-20.

13. Hansen JD, Vojtech LN, Laing KJ. Sensing disease and danger: a survey of vertebrate PRRs and their origins. Dev Comp Immunol. 2011;35(9):886-97.

14. Medzhitov R, Preston-Hurlburt P, Janeway CA Jr. A human homologue of the Drosophila Toll protein signals activation of adaptive immunity. $\mathrm{Na}$ ture. 1997;388(6640):394-7.

15. Fritz JH, Ferrero RL, Philpott DJ, Girardin SE. Nod-like proteins in immunity, inflammation and disease. Nat Immunol. 2006;7(12):1250-7.

16. Takahasi K, Yoneyama M, Nishihori T, Hirai R, Kumeta H, Narita R, et al. Nonself RNAsensing mechanism of RIG-I helicase and activation of antiviral immune responses. Mol Cell. 2008;29(4):428-40.

17. Janeway CA, Medzhitov R. Innate immune recognition. Ann Rev Immunol. 2002;20:197-216.

18. Guermonprez P, Valladeau J, Zitvogel L, Thery $\mathrm{C}$, Amigorena S. Antigen presentation and T cell stimulation by dendritic cells. Annu Rev Immunol. 2002;20:621-67.

19. Chan JL, Tang KC, Patel AP, Bonilla LM, Pierobon N, Ponzio NM, et al. Antigen-presenting property of mesenchymal stem cells occurs during a narrow window at low levels of interferon-gamma. Blood. 2006;107(12):4817-24. 
20. De Gregorio E, D’Oro U, Wack A. Immunology of TLR-independent vaccine adjuvants. Curr Opin Immunol. 2009;21(3):339-45.

21. Oleszycka E, Lavelle EC. Immunomodulatory properties of the vaccine adjuvant alum. Curr Opin Immunol. 2014;28:1-5.

22. García A, De Sanctis JB. An overview of adjuvant formulations and delivery systems. APMIS. 2014;122(4):257-67.

23. Seubert A, Monaci E, Pizza M, O’Hagan DT, Wack A. The adjuvants aluminumhydroxide and MF59 induce monocyte and granulocyte chemoattractants and enhance monocyte differentiation toward dendritic cells. J Immunol. 2008;180(8):5402-12

24. Eisenbarth SC, Colegio OR, O'Connor W, Sutterwala FS, Flavell RA. Crucial role for the Nalp3 inflammasome in the immunostimulatory properties of aluminium adjuvants. Nature. 2008;453(7198):1122-6.

25. Sharp FA, Ruane D, Claass B, Creagh E, Harris J, Malyala P, et al. Uptake of particulate vaccine adjuvants by dendritic cells activates the NALP3 inflammasome. Proc Natl Acad Sci U S A. 2009;106(3):870-5.

26. Kool M, Soullié T, van Nimwegen M, Willart MA, Muskens F, Jung S, et al. Alum adjuvant boosts adaptive immunity by inducing uric acid and activating inflammatory dendritic cells. J Exp Med. 2008;205(4):869-82.

27. Lambrecht BN, Kool M, Willart MA, Hammad H. Mechanism of action of clinically approved adjuvants. Curr Opin Immunol. 2009;21(1):23-9.

28. Hem SL, Johnston CT, HogenEsch H. Imject Alum is not aluminum hydroxide adjuvant or aluminum phosphate adjuvant. Vaccine. 2007;25(27):49856.

29. Hem SL. And Hogen Esch H. Relationship between physical and chemical properties of aluminium-containing adjuvants and immunopotetnation. Expert Rev. Vaccines. 2007;6(5):658-98.

30. Gołoś A, Lutyńska A. Aluminium-adjuvanted vaccines--a review of the current state of knowledge. Przegl Epidemiol. 2015;69(4):731-4

31. Fox CB, Haensler J. An update on safety and immunogenicity of vaccines containing emulsion-based adjuvants. Expert Rev Vaccines. 2013;12(7):74758.

32. Didierlaurent AM, Morel S, Lockman L, Giannini SL, Bisteau M, Carlsen H, et al. AS04, an alumi- num salt- and TLR4 agonist-based adjuvant system, induces a transient localized innate immune response leading to enhanced adaptive immunity. J Immunol. 2009;183(10):6186-97.

33. Bode C, Zhao G, Steinhagen F, Kinjo T, Klinman DM. CpG DNA as a vaccine adjuvant. Expert Rev Vaccines. 2011;10(4):499-511.

34. Longhi MP, Trumpfheller C, Idoyaga J, Caskey M, Matos I, Kluger C, et al. Dendritic cells require a systemic type I interferon response to mature and induce CD4+ Th1 immunity with poly IC as adjuvant. J Exp Med. 2009;206(7):1589-602.

35. Gorden KB, Gorski KS, Gibson SJ, Kedl RM, Kieper WC, et al. Synthetic TLR agonists reveal functional differences between human TLR7 and TLR8. J Immunol. 2005;174(3):1259-68.

36. Huleatt JW, Jacobs AR, Tang J, Desai P, Kopp EB, Huang Y, et al. Vaccination with recombinant fusion proteins incorporating Toll-like receptor ligands induces rapid cellular and humoral immunity. Vaccine. 2007;25(4):763-75.

37. Henriksen-Lacey M, Korsholm KS, Andersen P, Perrie Y, Christensen D. Liposomal vaccine delivery systems. Expert Opin Drug Deliv. 2011;8(4):50519.

38. Drane D, Gittleson C, Boyle J, Maraskovsky E. ISCOMATRIX adjuvant for prophylactic and therapeutic vaccines. Expert Rev Vaccines. 2007;6(5):761-72.

39. Ahmed SS, Plotkin SA, Black S, Coffman RL. Assessing the safety of adjuvanted vaccines. Sci Transl Med. 2011;3(93):93rv2.

40. Pellegrino P, Clementi E, Radice S. On vaccine's adjuvants and autoimmunity: Current evidence and future perspectives. Autoimmun Rev. 2015;14(10):880-8

41. De Vincenzo R, Conte C, Ricci C, Scambia G, Capelli G. Long-term efficacy and safety of human papillomavirus vaccination. Int J Womens Health. 2014;6:999-1010.

42. Stassijns J, Bollaerts K, Baay M, Verstraeten T. A systematic review and meta-analysis on the safety of newly adjuvanted vaccines among children. Vaccine. 2016;34(6):714-22.

43. Ahmed SS, Schur PH, MacDonald NE, Steinman L. Narcolepsy, 2009 A(H1N1)pandemic influenza, and pandemic influenza vaccinations: what is known and unknown about the neurological disorder, the role for autoimmunity, and vaccine adjuvants. J Autoimmun. 2014;50:1-11. 
44. Masoudi S, Ploen D, Kunz K, Hildt E. The adjuvant component $\alpha$-tocopherol triggers via modulation of Nrf2 the expression and turnover of hypocretin in vitro and its implication to the development of narcolepsy. Vaccine. 2014;32(25):2980-8.

45. Ahmed SS, Volkmuth W, Duca J, Corti L, Pallaoro M, Pezzicoli A, et al. Antibodies to influenza nucleoprotein cross-react with human hypocretin receptor 2. Sci Transl Med. 2015;7(294):294ra105.

46. Han F, Lin L, Warby SC, Faraco J, Li J, Dong SX, et al. Narcolepsy onset is seasonal and increased following the $2009 \mathrm{H} 1 \mathrm{~N} 1$ pandemic in China. Ann Neurol. 2011;70(3):410-7.

47. European Medicines Agency. Committee for Medicinal Products for Human Use (CHMP) Assessment report. Pandemrix. EMA/ CHMP/566359/2015. [updated 2016 Jun 10; cited 2017 Jan 10]. Available from: http://www.ema. europa.eu/ema/index.jsp?curl=pages/medicines $/$ human/medicines/000832/human_med_000965. jsp\&mid=WC0b01ac058001d124.

48. Rimaniol AC, Gras G, Verdier F, Capel F, Grigoriev VB, Porcheray F, et al. Aluminum hydroxide adjuvant induces macrophage differentiation towards a specialized antigen-presenting cell type. Vaccine. 2004;22(23-24):3127-35.

49. Israeli E, Agmon-Levin N, Blank M, Shoenfeld Y. Macrophagic myofaciitis a vaccine (alum) autoimmune-related disease. Clin Rev Allergy Immunol. 2011;41(2):163-8.

50. Cadusseau J, Ragunathan-Thangarajah N, Surenaud M, Hue S, Authier FJ, Gherardi RK. Selective elevation of circulating CCL2/MCP1 levels in patients with longstanding post-vaccinal macro- phagic myofasciitis and ASIA. Curr Med Chem. 2014;21(4):511-7.

51. World Health Organization. Statement from the Global Advisory Committee on Vaccine Safety on aluminium-containing vaccines [updated 2008 Oct 23; cited 2017 Jan 10]. Available from: http:// www.who.int/vaccine_safety/committee/topics/ aluminium/statement_112002/en/

52. Gronseth GS. Gulf war syndrome: a toxic exposure? A systematic review. Neurol Clin. 2005;23(2):52340.

53. Asa PB, Cao Y, Garry RF. Antibodies to squalene in Gulf War syndrome. Exp Mol Pathol. 2000;68(1):55-64.

54. Phillips CJ, Matyas GR, Hansen CJ, Alving CR, Smith TC, Ryan MA. Antibodies to squalene in US Navy Persian Gulf War veterans with chronic multisymptom illness. Vaccine. 2009;27(29):3921-6.

55. Lippi G, Targher G, Franchini M. Vaccination, squalene and anti-squalene antibodies: facts or fiction? Eur J Intern Med. 2010;21(2):70-3.

56. Guimarães LE, Baker B, Perricone C, Shoenfeld Y. Vaccines, adjuvants and autoimmunity. Pharmacol Res. 2015;100:190-209.

57. Shoenfeld Y, Agmon-Levin N. 'ASIA' - autoimmune/inflammatory syndrome induced by adjuvants. J Autoimmun. 2011;36(1):4-8.

58. Hawkes D, Benhamu J, Sidwell T, Miles R, Dunlop RA. Revisiting adverse reactions to vaccines: A critical appraisal of Autoimmune Syndrome Induced by Adjuvants (ASIA). J Autoimmun. 2015;59:77-84. 